\title{
La conservation au service du développement durable de tous les types de forêt dans le monde: Compendium des termes et notions ${ }^{1}$
}

\author{
Compilation de Paul L. Aird ${ }^{2}$ \\ Version française révisée par Michel Boudoux ${ }^{3}$
}

La conservation des forêts au niveau planétaire est devenue une priorité à l'échelle internationale dans les énoncés de politiques ainsi que dans les programmes politiques, scientifiques, environnementaux et commerciaux. Depuis la conférence des Nations Unies sur l'environnement et le développement durable tenue lors du Sommet de la Terre, à Rio de Janeiro en juin 1992, la question des forêts planétaires a pris de l'importance lors de nombreux forums, de négociations et de débats internationaux. Le fait de comprendre et d'interpréter de la même façon les termes se rapportant aux forêts et à la foresterie constitue la pierre angulaire nécessaire au maintien d'un dialogue international qui soit constructif et efficace en matière de forêts et de foresterie.

Les termes qui font l'objet du présent compendium sont employés couramment dans les conversations et les écrits d'ordre scientifique, technique ou général portant sur la conservation au service du développement durable de tous les types de forêt dans le monde. Nous les présentons ici avec leur définition et avec

${ }^{1}$ L'auteur remercie de leur précieuse collaboration les membres suivants du personnel de Ressources naturelles Canada, Service canadien des forêts : le $\mathrm{D}^{\mathrm{r}} \mathrm{J} . \mathrm{S}$. Maini, pour avoir proposé le présent travail et y avoir accordé son appui; les $\mathrm{D}^{\mathrm{rs}} \mathrm{T}$. Boyle and $\mathrm{O}$. Hendrickson, pour avoir révisé le manuscrit et présenté des suggestions pertinentes; ainsi que $\mathrm{M}$. Normand Lafrenière et le $\mathrm{D}^{\mathrm{r}}$ Michel Boudoux pour avoir supervisé la traduction du manuscrit; et à M. François Lavallée et à $\mathrm{M}^{\mathrm{me}}$ Francine Bertrand qui ont traduit le manuscrit en français et en espagnol respectivement. Des remerciements vont aussi à $\mathrm{M}$. Marc Sabourin, de la clinique de diagnostic phytosanitaire du ministère de l'agriculture, de l'alimentation et des affaires rurales de l'Ontario, et au $\mathrm{D}^{\text {re }}$ Isabel Bellocq, de la faculté de foresterie de l'université de Toronto, qui ont révisé les versions finales en français et en espagnol respectivement, ainsi qu'à de nombreux étudiants qui ont participé à la révision.

${ }^{2}$ Professeur, Conservation et Politiques Forestières, Faculty of Forestry, University of Toronto, Toronto (Ontario), Canada M5S 3B3

${ }^{3} \mathrm{D}^{\mathrm{r}}$ Michel Boudoux, directeur de la recherche, Forêts Canada - Région du Québec, Sainte-Foy (Québec), Canada. certaines explications afin de favoriser une uniformisation nationale et internationale du sens qu'on leur prête.

Le sens d'un mot comporte souvent plusieurs nuances, ce qui peut entraîner des erreurs dans l'usage. Nous espérons que cette liste de termes spécialisés favorisera le développement, l'utilisation et la compréhension d'une terminologie uniforme tout en décourageant l'emploi de termes ambigus.

Pour certains termes, comme pluie acide, les définitions abondent. Nous les avons étudiées minutieusement avant de les choisir et de les adapter pour présenter ce qui paraît être la formule la plus appropriée et la plus détaillée pour un usage normalisé. Dans certains cas, on trouvera des précisions supplémentaires ainsi que de nouvelles définitions.

Nous n'avons pas eu recours aux définitions des dictionnaires courants, parfois à cause de leur caractère désuet, souvent parce qu'elles ne concernaient pas précisément la conservation de la forêt et enfin, dans certains cas, par respect du droit d'auteur. Ainsi, la compilation de ce glossaire a demandé la consultation d'un grand nombre de sources spécialisées.

Quelques unes des définitions ont été écrites par l'auteur de ce glossaire. Toute autre définition est suivie de la source qui l'a inspirée; la liste des références se trouve à la fin du document.

Précisons que plusieurs des définitions présentées ne constituent pas des citations exactes. Notre travail a consisté principalement à sélectionner la nomenclature des termes liés à la conservation de la forêt et à copier, réviser, réécrire ou rédiger les définitions afin de clarifier leur sens et leur usage dans la collectivité internationale.

Les commentaires et suggestions visant à améliorer cette compilation, que ce soit sous forme d'ajouts, de suppressions ou de révisions, seront les bienvenus. Ainsi arrivera-t-on peut-être à un consensus universel sur les termes à utiliser en matière de conservation de la forêt.

\section{Termes et notions concernant la conservation des forêts (avec la traduction respective des termes en anglais et en espagnol)}

abiotique (abiotic, abiótico) caractérisé par l'absence de vie; qui concerne les éléments inanimés de l'environnement

accumulation biologique (bioaccumulation) (biological accumulation (bioaccumulation), acumulación biológica (bioacumulación)) également appelée "amplification biologique". Augmentation graduelle de la concentration d'une substance persistante dans un organisme à mesure qu'il vieillit ou dans une suite d'organismes, depuis les producteurs primaires jusqu'aux consommateurs primaires et secondaires

agroforesterie (agroforestry, agrosilvicultura) terme général désignant les régimes d'aménagement qui consistent à intégrer délibérément les plantes vivaces ligneuses à la culture de la terre, à l'élevage ou aux deux sur une même unité d'aménagement du territoire. L'intégration peut prendre la forme d'une coexistence simultanée ou d'une alternance. Il existe normalement des interactions écologiques et économiques entre les éléments ligneux et non ligneux en agroforesterie (Conseil international pour la recherche en agroforesterie 1991)

amélioration des arbres (tree improvement, mejora de los árboles) terme mal choisi qui désigne la sélection, l'évaluation et l'amélioration génétique des arbres ayant pour but de leur donner des caractéristiques recherchées comme une crois- 
sance rapide et une résistance à la sécheresse, à la maladie ou aux insectes

aménagement à rendement soutenu (sustained-yield management, manejo con rendimiento sostenido) aménagement visant à réaliser le plus tôt possible un équilibre entre la croissance et l'abattage (Society of American Foresters 1971, 1983)

aménagement de la faune (wildlife management, manejo de la fauna silvestre) application de principes scientifiques et techniques au maintien des populations et aux habitats d'espèces sauvages (en particulier les mammifères, les oiseaux et les poissons), essentiellement à des fins récréatives ou scientifiques. Ce terme comprend la notion plus étroite de gestion du gibier, qui inclut des objectifs semi-commerciaux, c.-à-d. la récolte limitée de gibier sauvage (Society of American Foresters 1971, 1983)

aménagement de l'écosystème (ecosystem management, manejo del ecosistema) ensemble de mesures prises par l'être humain qui consistent à sélectionner, à planifier, à organiser et à mettre en oeuvre des programmes conçus pour réaliser des objectifs déterminés à l'échelle de l'écosystème; il peut s'agir de mesures de protection visant à ce que la nature ne soit pas dérangée par l'influence humaine ou encore d'interventions plus actives nécessaires au maintien de la diversité, à la mise en place d'installations, à la limitation des populations ou à l'élimination d'espèces exotiques (McNeely et autres 1990)

aménagement durable (sustainable management, manejo sostenible) gestion de l'utilisation, de l'exploitation et de la protection des ressources naturelles et physiques d'une manière ou à un rythme qui permettent aux personnes et aux collectivités d'assurer leur bien-être social, économique et culturel ainsi que de préserver leur santé et leur sécurité, a) tout en préservant l'aptitude des ressources naturelles et physiques (à l'exclusion des minéraux) à répondre aux besoins raisonnablement prévisibles des générations futures, b) tout en sauvegardant l'aptitude de l'air, de l'eau, du sol et des écosystèmes à entretenir la vie et c) tout en évitant, en corrigeant ou en atténuant les effets pervers des activités sur l'environnement (New Zealand Government 1991)

aménagement durable des forêts (sustainable forest management, manejo forestal sostenible) régime d'aménagement d'un territoire forestier qui vise à maintenir les capacités de production et de renouvellement de même que la diversité génétique, spécifique et écologique des écosystèmes forestiers. L'aménagement forestier durable est nécessaire au développement durable des forêts (Maini 1991)

aménagement forestier (forest management, manejo forestal) d'une manière générale, application des principes scientifiques, économiques et sociaux à l'administration et à l'exploitation d'une forêt à des fins déterminées; plus particulièrement, branche de la foresterie qui s'intéresse aux questions administratives, économiques, juridiques et sociales globales ainsi qu'aux activités essentiellement scientifiques et techniques, notamment la sylviculture, la protection et la réglementation de la forêt (Society of American Foresters 1971, 1983)

aménagement intégré des ressources (integrated resource management, manejo integrado de recursos) aménagement commun d'au moins deux ressources naturelles (eau, sol, bois, pâturage, faune, loisirs, etc.) intégrant les valeurs et les intérêts de la com- munauté planétaire dans la conception, la planification et la mise en oeuvre de principes, de programmes et de projets visant à exploiter et à préserver ces ressources à perpétuité (Mitchell 1986, Peel 1991)

analyse de vulnérabilité (de viabilité) d'une population (population vulnerability (viability) analysis, análisis de vulnerabilidad (de viabilidad) de una población) méthode d'analyse visant à évaluer la taille minimale nécessaire à une population d'une espèce pour préserver son existence (Gilpin et Soulé 1986)

arbre (tree, árbol) plante ligneuse vivace comportant un tronc, ou fût, distinct du houppier et se terminant en ramifications et en feuillage; comprend aussi les grands arbustes, les bambous, les cannes, etc. (Society of American Foresters 1971, 1983)

banque de gènes (gene bank, banco de genos) installations comme les banques de graines ou de sperme mises en place pour la conservation ex situ des tissus ou cellules reproducteurs d'organismes (World Resources Institute et autres, 1992).

bassin hydrographique (watershed, cuenca hidrográfica) région drainée par un cours d'eau ou un réseau de cours d'eau souterrains ou superficiels

bioénergie (bio-energy, bionergía) énergie cinétique libérée par la biomasse lorsqu'elle est consommée, brûlée ou convertie en combustible, ou énergie potentielle comprise dans la biomasse (Commission des communautés européennes 1983, Hendrickson 1993)

biologie de la conservation (conservation biology, biología de la conservatión) discipline qui traite du contenu de la biodiversité, des processus naturels qui la produise ainsi que des techniques utilisées pour la maintenir malgré les perturbations de l'environnement causées par l'homme (Wilson, 1992)

biomasse (biomass, biomasa) poids (ou équivalent estimé) de la matière organique, y compris les animaux, les plantes et les micro-organismes, vivants ou morts, au-dessus ou au-dessous de la surface terrestre (Aird 1979)

biome (biome, bioma) vaste ensemble complexe d'organismes partageant le même milieu (ex. la forêt résineuse boréale), conditionné par le climat et le sol (Tansley 1935)

biosphère (biosphere, biosfera) partie de la terre et de l'atmosphère où peuvent subsister des organismes vivants (Australie, Resource Assessment Commission 1991)

biote (biota, biota) ensemble des organismes se trouvant dans une région donnée (Keystone Center 1991)

biotique (biotic, biótico) qui relève de la vie; qui se rapporte à l'élément animé de l'environnement

boisement (afforestation, forestación) action de garnir d'arbres, par semence, par plantation ou par régénération naturelle, un terrain dont les arbres sont absents depuis toujours ou depuis longtemps (Society of American Foresters 1971, 1983)

capacité de charge (carrying capacity [1], carga cinegética (capacidad de carga)) voir "capacité récréative" et "possibilité de charge". Nombre (ou poids) des organismes d'une espèce et de qualités données, qui peuvent survivre dans un écosystème donné, sans en entraîner la détérioration, dans les condi- 
tions de milieu les plus défavorables susceptibles de se produire au cours d'un intervalle de temps donné (Society of American Foresters, 1971, 1977, 1983; Republic of the Philippines 1982)

capacité récréative (carrying capacity 3, capacidad de alojamiento) nombre maximum d'utilisateurs que peut supporter une zone récréative sans détérioration qualitative des loisirs ni des ressources (Society of American Foresters, 1971, 1977, 1983; Republic of the Philippines 1982)

carbone voir "réservoir à carbone" et "puits à carbone"

clone (clone, clon) individu ou groupe d'individus né d'un même organisme par multiplication asexuelleuement identique au parent

communauté (community, comunidad) groupe intégré d'espèces habitant une région donnée et dont chacune influe sur la répartition, l'abondance et l'évolution des autres (World Resources Institute et autres 1992)

conservation (conservation, conservación) gestion de l'utilisation que fait l'humain de la biosphère visant à ce que les générations actuelles en tirent le maximum d'avantages durables sans amoindrir sa capacité de répondre aux besoins et aux aspirations des générations futures, ce qui comprend la préservation, l'entretien, l'utilisation durable, le rétablissement et l'amélioration du milieu naturel. La théorie moderne de la conservation ajoute que les efforts de conservation ne visent pas tant l'état physique d'un système écologique que les processus écologiques qui donnent naissance à cet état et l'entretiennent. (Australie, Resource Assessment Commission 1991). La conservation vise autant les forêts consacrées à l'exploitation que les réserves écologiques, bien que les objectifs d'aménagement diffèrent (Maini et Carlisle 1974)

contaminer (contaminate, contaminar) rendre impur en mettant en contact ou en mélangeant avec une substance étrangère ou nocive; altérer, infecter, empoisonner, polluer

couvert forestier (forest cover, cubierta forestal) ensemble des arbres et des autres plantes qui occupent le sol d'une forêt, y compris le tapis végétal (Society of American Foresters 1971, 1983)

croissance durable (sustainable growth, crecimiento sostenible) "croissance durable" est un terme contradictoire: rien de physique ne peut croître indéfiniment (Alliance mondiale pour la nature et autres 1991)

culture (culture, cultura) ensemble des modes de vie représentés par un groupe d'être humains et transmis de génération en génération (Australie, Resource Assessment Commission 1991)

déboisement (deforestation, desforestación) action de supprimer la forêt durablement pour une utilisation non forestière. La coupe à blanc (même avec dessouchage), si elle est suivie après peu d'un reboisement à des fins forestières, n'est pas du déboisement (Society of American Foresters 1971, 1983)

dégradation (degradation, degradación) diminution de la productivité ou de la diversité biologiques (Sargent et Lowcock 1991). Réduction du rendement, de la qualité, etc.

dépôt acide (ucidic deposition, depósito ácido) phénomène par lequel les acides sont incorporés à la pluie, dans la neige, etc., ("dépôt humide"), ou par lequel les cendres volantes, les sulfates et nitrates et les gaz comme l'anhydride sulfureux et l'oxyde d'azote sont déposés ou adsorbés sur une surface ("dépôt sec"). Les particules sèches et les gaz peuvent se transformer en acides après leur dépôt ou leur adsorption si elles entrent en contact avec l'eau (Chambre des Communes du Canada 1981)

dépression de consanguinité (inbreeding depression, depresión por consanguinidad) diminution de la variabilité génétique due à l'accouplement de spécimens apparentés à un degré élevé (Baker 1989)

dérive génétique (genetic drift, deriva genética) la sélection aléatoire des gènes transmis d'une génération à la suivante tend à amoindrir la variation génétique, appelée dérive génétique, plus rapidement dans les populations restreintes que dans les populations nombreuses (Lacy 1987)

développement (development, desarrollo) progrès de l'aménagement et de l'utilisation des ressources naturelles visant à satisfaire les besoins des être humains et à améliorer la qualité de leur vie. Pour assurer la pérennité du développement, il faut tenir compte des facteurs sociaux et écologiques, ainsi que des facteurs économiques, de la base des ressources vivantes et non vivantes, des avantages et désavantages à court et à long terme ainsi que des solutions de rechange (Union internationale pour la conservation de la nature et de ses ressources 1980)

développement durable ou écodéveloppement (sustainable development, desarrollo sostenible) développement qui répond aux besoins du présent sans compromettre la possibilité pour les générations à venir de satisfaire les leurs. Le développement durable vise à satisfaire les besoins fondamentaux de tous et à fournir à chacun la possibilité de vivre une vie meilleure selon ses aspirations. Plutôt qu'un état d'harmonie immuable, c'est un processus évolutif dans lequel l'exploitation des ressources et leur renouvellement, l'orientation des investissements, le sens du progrès technologique et l'évolution des institutions tiennent compte des besoins futurs autant que des besoins présents (Commission mondiale de l'environnement et du développement 1988). Voir "développement soutenu"

développement durable des forêts (sustainable forest development, desarrollo forestal sostenible) développement comportant le maintien, sans dégradation inacceptable, du potentiel de production et de renouvellement et de la diversité génétique, spécifique et écologique des écosystèmes forestiers (Maini 1989)

développement soutenu (sustained development, desarrollo sostenido) développement maintenu, souvent d'une manière trop peu sensible aux contraintes sociales ou écologiques. Voir "développement durable"

disparu (extinct, extinguido) qui n'existe plus (Keystone Center 1991)

diversité biologique (biodiversité) (biological diversity (biodiversity), diversidad biológica (biodiversidad)) variété et variabilité observées chez les organismes vivants dans tous les écosystèmes, terrestres, marins ou d'eau douce, ainsi qu'entre les ensembles écologiques qu'ils habitent. La biodiversité existe à l'intérieur d'une espèce, entre les espèces ou dans un 
écosystème (Programme des Nations Unies pour l'environnement 1992)

diversité écosystémique (ecosystem diversity, diversidad ecosistémica) variété et variabilité des habitats, des communautés biotiques, des éléments abiotiques et des phénomènes écologiques existant dans une région donnée (McNeely et autres 1990)

diversité génétique (genetic diversity, diversidad genética) variation de la composition génétique des individus à l'intérieur d'une espèce, d'une variété ou d'une race ou entre plusieurs espèces, variétés ou races; variation génétique transmissible dans une population ou entre populations (World Resources Institute et autres 1992)

diversité spécifique (species diversity, diversidad específica) expression faisant référence à la pluralité des organismes vivants sur terre; on estime que le nombre d'espèces s'élève entre 5 et 100 millions et plus, quoique seulement 1,4 million, environ, a été décrit (McNeely et autres 1990). La diversité spécifique se mesure au nombre des espèces, aux populations et à leur répartition dans une unité géographique donnée. On peut la considérer à trois niveaux: la diversité microcosmique (type alpha), qui tient compte d'un même habitat, la diversité intercosmique (type bêta), qui tient compte de changements attribuables aux différences de topographie ou de climat selon les habitats, et la diversité macrocosmique (type gamma), qui concerne une grande superficie comprenant de nombreux habitats (Cody 1986, Tho 1991). Voir aussi "richesse spécifique" et "diversité taxonomique"

diversité culturelle (cultural diversity, diversidad cultural) variété et variabilité des structures sociales, systèmes de pensée et stratégies de l'être humain qui entrent en jeu lorsque ce dernier s'adapte aux situations et aux changements d'ordre biologique dans les différentes parties du monde (World Resources Institute et autres 1992)

\section{diversité écosystémique voir "diversité biologique"}

diversité génétique voir "diversité biologique"

diversité spécifique voir "diversité biologique"

diversité taxonomique (taxonomic diversity, diversidad taxonómica) mesure de la diversité spécifique tenant compte du degré de parenté. Par exemple, la terre compte beaucoup plus d'espèces que la mer, mais les espèces terrestres sont apparentées à un degré beaucoup plus élevé que les espèces océaniques. Ainsi, la diversité des écosystèmes marins est plus grande que ne le laisserait croire le simple dénombrement des espèces (World Resources Institute et autres 1991)

domestiquer (domesticate, domesticar) s'occuper d'un organisme sauvage, ou encore le cultiver ou l'apprivoiser

droits de coupe (stumpage, canon) prix exigé en retour du droit de couper du bois sur une terre forestière domaniale ou privée

écologie (ecology, ecología) branche de la science ayant pour objet l'étude des relations des organismes vivants entre eux et avec leur milieu (McNeely et autres 1990)

écologie forestière (silvics, ecología forestal) étude du cycle de vie, des besoins et des caractéristiques générales des arbres et peuplements forestiers en relation avec l'environnement et avec la pratique de la sylviculture (Society of American Foresters 1989)

écosystème (ecosystem, ecosistema) a l'origine, d'après A.G. Tansley (1935), tout ensemble complexe d'organismes vivants, avec leur milieu, que "nous isolons mentalement afin de l'étudier". Le terme comprend tous les organismes qui vivent ensemble ainsi que le milieu physique où ils se trouvent (Society of American Foresters 1971, 1983; Keystone Center 1991)

écosystème forestier (forest ecosystem, ecosistema forestal) système écologique composé d'éléments biotiques et abiotiques de l'environnement qui s'influencent les uns les autres et où le couvert des arbres représente plus de $20 \%$ de la superficie totale (Maini 1991)

endémique (endemic, endémica) voir "espèce"

environnement (environment, medio ambiente) ensemble des éléments biotiques et abiotiques d'un lieu qui influent sur le développement de la vie (Empire Forestry Association 1953)

épuisement (depletion, agotamiento) réduction graduelle ou destruction de ressources naturelles ou de sources d'énergie, d'argent, de produits, etc.

équilibre écologique (ecological balance, equilibrio ecológi$\mathrm{co}$ ) situation dynamique caractéristique d'une communauté d'organismes dont la diversité génétique, spécifique et écosystémique demeure relativement stable, quoiqu'elle puisse évoluer graduellement par succession naturelle

espèce (species, especie) population ou série de populations d'organismes pouvant s'accoupler librement les uns avec les autres mais non avec des membres d'autres espèces (Keystone Center 1991)

espèce endémique (espèce indigène) (endemic species (indigenous species), especie endémica (especie indígena)) espèce provenant d'une région particulière ou lui appartenant. Les adjectifs "endémique" et "indigène" sont préférés à "autochtone"

espèce indigène voir "espèce: espèce endémique"

espèce exotique (exotic species, especie exótica) espèce introduite d'une manière fortuite ou à dessein dans une région extérieure à son aire naturelle de répartition. L'adjectif "exotique" est préféré à "non autochtone"

sous-espèce (subspecies, subespecie) subdivision d'une espèce; population ou ensemble de populations occupant une zone précise et différant génétiquement des autres sous-espèces de la même espèce (World Resources Institute et autres 1992)

espèce indicatrice (indicator species, especie indicadora) espèce végétale, animale ou microbienne présentant des traits ou des réactions caractéristiques à un lieu, à un habitat, à un écosystème ou à une condition environnementale

espèces sauvages (wildlife, flora y fauna silvestres) Ensemble des mammifères, oiseaux, reptiles, batraciens, poissons, invertébrés, plantes, champignons, algues, bactéries et autres organismes vivant dans la nature (Groupe de travail sur la faune 
de l'Ontario 1991) Organismes non humaines, non domestiqués et non cultivés

étendue naturelle (wilderness, zona natural) partie du paysage naturel assez grande et variée pour constituer une unité écologique plus ou moins autorégulatrice, où l'interaction de l'homme avec le territoire et les communautés naturelles de plantes et d'animaux est minime et à laquelle la beauté et le caractère du paysage confèrent une valeur esthétique, culturelle et scientifique (Pimlott 1971)

exotique (exotic, exótica) voir "espèces"

explosion écologique (écoexplosion) (ecological explosion (ecoexplosion), explosión ecológica (ecoexplosión)) augmentation extraordinaire de la population d'un organisme vivant; par exemple, multiplication d'un virus infectieux comme celui de la grippe, d'une bactérie comme celle de la peste bubonique, d'un champignon comme celui de la maladie de la pomme de terre, d'une plante verte comme la poire cactus ou d'un animal comme l'écureuil gris (Elton 1957)

extinction (extinction, extinción) disparition d'une espèce, causée par l'absence de reproduction et par la mort de tous ses représentants (McNeely et autres 1990)

risque d'extinction (extinction risk, riesgo de extinción) on peut définir le risque d'extinction prématurée en énonçant les probabilités d'extinction dans un délai donné. Ces probabilités se fondent sur la théorie des délais d'extinction pour une population particulière et sur les échéances utiles pour les programmes de conservation. On compte trois catégories basées sur la diminution des risques d'extinction et sur l'augmentation des délais

espèce critique: probabilité d'extinction en 5 ans, ou deux générations au maximum, de $50 \%$;

espèce menacée d'extinction: probabilité d'extinction en 20 ans, ou dix générations au maximum, de $20 \%$;

espèce vulnérable: probabilité d'extinction en 100 ans de 10 \% (Mace et Lande 1991)

extirpation (extirpation, extirpación) extinction locale; fait pour une espèce ou une sous-espèce de disparaître d'un lieu ou d'une région sans s'éteindre dans toute son aire de distribution (McNeely et autres 1990)

ferme planétaire ( lobal farm, granja global) notion née de celle du village planétaire afin de souligner l'interdépendance des gens, des nations et de la nature. Grâce à la vitesse des échanges d'information, on peut aujourd'hui aménager, utiliser et partager avec autrui son patrimoine biologique. La responsabilité commune de préserver la diversité et la productivité biologiques du monde afin d'éliminer les injustices pouvant être subies par une génération par rapport aux autres pour toutes les espèces recrée le monde à l'image d'une ferme familiale planétaire. Le jardin planétaire et la forêt planétaire font partie intégrante de la ferme planétaire (Aird 1989)

foresterie (forestry, dasonomía) d'une manière générale, profession faisant appel à la science et à un savoir-faire particulier afin de créer, de conserver et d'aménager des forêts et des terres forestières de manière à permettre une utilisation permanente de leurs ressources matérielles et récréatives. Science, art et pratique de l'aménagement et de l'utilisation, au profit à longterme des humans, des ressources naturelles qui se trouvent sur les terres forestières ou y sont associées (Society of American Foresters 1971, 1983)

foresterie urbaine (urban forestry, silvicultura y arboricultura urbana) culture et aménagement d'arbres pour leur apport actuel et potentiel au bien-être physiologique, sociologique et économique d'une société urbaine (Jorgensen 1986)

forêt (forest, bosque) écosystème composé surtout d'arbres et de végétation ligneuse qui croissent ensemble de manière plus ou moins dense (Society of American Foresters 1971, 1983). Paysage dominé par des arbres et dont le couvert représente au moins $20 \%$ de la superficie totale (Maini 1991). Comprend les catégories suivantes:

forêt ancienne (old-growth forest, rodal maduro) forêt dominée par des organismes mûrs nés naturellement des organismes endémiques de la forêt ou de ses environs et sur laquelle l'activité humaine n'a pas eu d'effet majeur

forêt de production (production forest, bosque de producción) forêt aménagée de manière à obtenir des produits forestiers tout en préservant la bioproductivité de l'ensemble. La diversité biotique ou abiotique de la forêt peut être modifiée par labourage, par plantation et par culture d'espèces choisies, y compris l'introduction de variétés exotiques ou à croissance rapide

forêt naturelle (natural forest, bosque natural) forêt qui a évolué et s'est renouvelée naturellement à partir d'organismes qui étaient déjà sur place et sur laquelle l'activité humaine n'a pas eu d'effet majeur. Comprend entre autres les "vieilles forêts"

forêt patrimoniale (heritage forest, bosque patrimonial) forêt aménagée de manière à obtenir des produits forestiers tout en préservant le système naturel, y compris sa bioproductivité et sa diversité biotique et abiotique. L'abattage, le reboisement et les soins culturaux peuvent comprendre le recours aux techniques, aux équipements et aux méthodes modernes; les principaux efforts portent cependant sur le reboisement naturel aidé de l'implantation artificielle d'espèces endémiques appropriées

forêt permanente (permanent forest, bosque permanente) forêt désignée en vertu d'une politique d'utilisation des terres comme devant conserver son couvert en permanence, c'est-à-dire comme ne devant servir à aucun autre type d'utilisation (Maini et Ullsten 1991)

forêt rurale (farm forest/rural forest, bosque rural) arbres, haies, brise-vent, vergers, plantations, forêts et organismes associés se trouvant sur des terres agricoles ou des pâturages

forêt tampon (buffer forest, bosque tampón) forêt servant de terrain de transition entre deux zones aménagées à des fins différentes: par exemple, forêt séparant un parc national d'une aire de culture et aménagée de manière à pouvoir protéger la valeur du parc tout en fournissant les produits forestiers nécessaires à la communauté rurale

forêt traditionnelle (traditional forest, bosque tradicional) forêt qui se perpétue naturellement et en permanence grâce aux organismes qui y sont déjà établis et que l'on exploite 
par les méthodes traditionnelles conformément à des usages ayant cours depuis longtemps afin de répondre à des besoins locaux

forêt urbaine (urban forest, bosque urbano) arbres, forêts et organismes associés qui croissent près de bâtiments et dans des jardins, des espaces verts, des parcs et des terrains de golf, dans un village, une ville ou une banlieue

gaule (sapling, brinzal) terme général désignant un jeune arbre qui n'est plus un semis sans être encore un arbre adulte, c.-à-d. qui mesure plus d'un mètre de haut environ et approximativement 2,5 à $10 \mathrm{~cm}$ de diamètre (Society of American Foresters 1971, 1983)

gène (gene, gen) unité fonctionnelle de l'hérédité; partie de la molécule d'acide désoxyribonucléique ( $\mathrm{ADN}$ ) encodant un enzyme unique ou une unité protéinique structurelle unique (World Resources Institute et autres 1992)

habitants des forêts (forest dwellers, habitantes del bosque) personnes qui vivent dans un écosystème forestier et qui en ont besoin pour conserver leur mode de vie, leurs structures sociales, leur identité culturelle et leurs connaissances traditionnelles, qui doivent être sauvegardés et respectés (Maini 1991). Le terme peut aussi comprendre "les personnes et les autres organismes" comme habitants des forêts

habitat (habitat, hábitat) milieu naturel où vit un organisme ou une population. Le terme peut désigner tous les organismes et leur milieu physique à un endroit particulier (Ministère de l'Agriculture du Canada 1976; Keystone Center 1991)

indigène voir "espèce"

industrie extractive (resource industry, industria extractiva) industrie reposant sur les ressources primaires tirées de l'agriculture, de la pêche ou de l'exploitation des forêts ou des mines, par exemple le blé, la morue, le pin ou le minerai de fer

intégrité écologique (d'un écosystème) (ecological (ecosystem) integrity, integridad ecológica (de un ecosistema)) qualité d'un écosystème naturel aménagé ou non où se produisent des phénomènes écologiques naturels et où la diversité génétique, spécifique et écosystémique est assurée pour l'avenir (Service canadien des parcs 1991)

intendance (stewardship, administración) la science, l'art, et le savoir-faire d'une gestion des resources responsable

intendant (steward, administrador) personne responsable de la surveillance et de la gestion de ressources

maintenir (sustain, mantener) fournir en permanence les nécessités de la vie; tenir en vie; préserver la qualité, la quantité, la productivité, la biodiversité, etc.

marchand (merchantable, comerciable) se dit de la partie d'un arbre ou d'une récolte qui est commercialisable dans des conditions économiques données (Empire Forestry Association 1953)

monoculture (monoculture, monocultivo) sélection et culture, par semence, plantation ou amélioration, d'une espèce, d'une variété, d'une souche génétique ou d'un clone seulement, souvent accompagnées de la destruction ou de l'exclusion des autres niche (niche, nicho) milieu unique permettant l'existence d'un organisme ou d'une espèce

organisme (organism, organismo) tout être vivant composé d'une cellule ou plus

parc voir "zone protégée"

patrimoine (heritage, patrimonio) ce dont les personnes ou les collectivités peuvent hériter et ce qu'elles peuvent transmettre à leurs successeurs

patrimoine culturel (cultural heritage, patrimonio cultural) coutumes, traditions, littérature, art, collections de musée, bâtiments historiques, sentiers, routes (Ministère de la Culture et des Communications de l'Ontario 1990)

patrimoine naturel (natural heritage, patrimonio natural) terre, eau, air, roches, fossiles, plantes, animaux, microorganismes, habitats et écosystèmes

pesticide (pesticide, pesticida) produit naturel ou artificiel servant à gêner, à supprimer ou à tuer un organisme jugé nuisible pour la croissance, le développement ou la survie d'autres organismes; comprend les acaricides, les arboricides, les bactéricides, les biocides, les fongicides, les herbicides, les insecticides, les miticides, les nématicides, les piscicides et les virucides

peuplement (stand, rodal) groupe continu de plantes suffisamment uniforme du point de vue de la répartition des âges et des tailles, de la composition, de la structure, de la qualité de l'endroit ou de l'emplacement pour constituer une unité distincte (Society of American Foresters 1989)

plantation (plantation, plantación) peuplement de plantes en croissance aménagé par l'homme, soit par semence, soit par plantation

pluie acide (acid rain, lluvia ácida) pluie dont le $\mathrm{pH}$ est inférieur à 5,6. (Chambre des Communes du Canada 1981). Souvent utilisé comme synonyme de "dépôt acide" ou de "précipitation acide", équivalence impropre à strictement parler

polluer (pollute, contaminar) contaminer la terre, l'eau, l'air, la végétation, l'environnement, etc., avec des substances jugées nocives pour la santé des organismes vivants

possibilité de charge (carrying capacity 2, carga) population maximale de bétail que peut supporter un terrain de parcours déterminé (ou un terrain donné occupé par des animaux sauvages) sans diminution des ressources fourragères ni en quantité, ni en qualité, non plus que de la valeur des autres ressources attachées au même terrain, y compris le sol, la végétation et le bassin versant (Society of American Foresters, 1971, 1977, 1983; Republic of the Philippines 1982)

précipitation acide (acidic precipitation, precipitación ácida) pluie, neige, neige fondante, grêle ou brouillard, généralement dont le $\mathrm{pH}$ est inférieur à 5,6. (L'échelle du pH varie de zéro, qui représente l'acidité maximale, à 14 , qui représente une acidité nulle; un $\mathrm{pH}$ de 7 caractérise une solution neutre. Présentant un $\mathrm{pH}$ de 5,6, la pluie normale est un peu acide à cause des réactions chimiques qui se produisent avec le gaz carbonique dans l'atmosphère terrestre.) Les précipitations acides résultent principalement de l'émission gazeuse d'oxydes de soufre 
et d'oxydes d'azote qui se transforment respectivement en acide sulfurique et en acide nitrique au cours d'un transport atmosphérique pouvant couvrir des centaines ou des milliers de kilomètres (Chambre des Communes du Canada 1981)

préservation (preservation, preservación) action de protéger une partie du milieu naturel contre un facteur de perturbation qui n'est pas naturel. La préservation ne consiste pas nécessairement à maintenir une zone dans un état donné, car les phénomènes naturels écologiques et autres continueront. La préservation fait partie de la conservation et ne s'y oppose pas

productivité biologique (bioproductivité) (biological productivity (bioproductivity), productividad biológica (bioproductividad)) capacité de produire la biomasse; production de biomasse

puits à carbone (sink, carbon; pozo de carbono) zone où la consommation de carbone par les organismes vivants dépasse le taux de libération de carbone, de sorte que cet élément est activement séquestré dans des formes organiques ou inorganiques (Hendrickson 1993)

reboisement (reforestation, reforestación) action de regarnir d'arbres une terre forestière (Society of American Foresters 1971, 1983)

reboiser (reforest, reforestar) regarnir d'arbres une terre forestière ancienne ou récente par des moyens naturels ou artificiels

régénération artificielle (regeneration, artificial; regeneración artificial) renouvellement d'un peuplement par semence directe ou par plantation de semis ou de boutures (Society of American Foresters 1971, 1983)

régénération naturelle (regeneration, natural; regeneración natural) renouvellement d'un peuplement par semence naturelle, par drageonnement ou par marcottage (Society of American Foresters 1989)

rendement soutenu (sustained yield, rendimiento sostenido) production pouvant être tirée d'une culture ou d'une population en permanence selon une intensité de gestion donnée (Society of American Foresters 1971, 1983)

réserve voir "zone protégée"

réservoir à carbone (reservoir, carbon; depósito de carbono) zone où s'est accumulé du carbone en quantité appréciable en raison de l'activité passée ou présente d'un puits à carbone (Hendrickson 1993)

réservoir de gènes (gene pool, reservorio de genes) tous les gènes d'une espèce, d'une sous-espèce, ou d'un groupe d'organismes se reproduisant entre-eux

ressource (resource, recurso) tout ce qui est ou peut s'avérer utile à des personnes ou à d'autres organismes: nourriture, combustible, bien, forêt, minéraux et argent

ressource biologique (biological resource, recurso biológico) comprend les ressources génétiques, les organismes ou les parties d'organismes, les populations et tout autre élément biotique d'un écosystème présentant une valeur réelle ou potentielle pour l'humanité (Programme des Nations Unies pour l'environnement 1992) ou pour d'autres organismes ressource naturelle ou richesse naturelle (natural resource, recurso natural) ressource renouvelable (forêt, eau, faune, sol, etc.) ou non renouvelable (pétrole, charbon, minerai de fer, etc.) prodiguée par la nature (McNeely et autres 1990). Voir "industrie extractive"

restauration (restoration, restauración) retour d'un écosystème ou d'un habitat à son organisation collective d'origine, à la complémentarité naturelle de ses espèces et à ses fonctions naturelles (World Resources Institute et autres 1992)

risque d'extinction voir "extinction"

richesse spécifique (species richness, riqueza específica) ensemble des espèces se trouvant dans une aire donnée, sans égard à la répartition (Tho 1991)

rotation (rotation, rotación) cycle de régénération, de développement et de récolte d'une espèce (ex. pin gris) ou d'une suite d'espèces (ex. maïs, avoine, trèfle); utilisation de différentes parties d'une zone en succession (pour la chasse, le pâturage, etc.)

spirale d'extinction (extinction vortex, espiral de extinción) phénomènes génétiques et démographiques qui entrent en jeu lorsqu'une population devient peu nombreuse et isolée et que ses représentants se mettent à interagir d'une manière qui crée ce que l'on a appelé la "spirale d'extinction". L'érosion génétique due à l'endogamie et les difficultés d'adaptation peuvent diminuer davantage une population déjà réduite, ce qui rend d'autant plus incertaines les possibilités de reproduction, accentue la dépopulation, accroît le degré de consanguinité et amoindrit la diversité génétique. La population se dirige ainsi inéluctablement vers l'extinction à un rythme sans cesse accéléré (Gilpin et Soulé 1986; Lacy et autres 1992)

stratégie mondiale de la conservation (world conservation strategy, estrategia mundial de conservación) ensemble de stratégies internationales, nationales et régionales conçues pour atteindre les trois grands objectifs de conservation des ressources vivantes (Union internationale pour la conservation de la nature et de ses ressources 1980):

maintenir les processus écologiques essentiels et les systèmes entretenant la vie (régénération et protection du sol, recyclage des nutriments, épuration naturelle des eaux) dont dépendent la survie et le développement de l'humanité;

préserver la diversité génétique (gamme de matériel génétique existant dans les organismes du monde), dont dépendent le fonctionnement d'un grand nombre de ces processus et systèmes, les programmes de sélection nécessaires à la protection et à l'amélioration des plantes cultivées, des animaux domestiques et des micro-organismes ainsi qu'une bonne part des progrès scientifiques et médicaux, des innovations techniques et de l'avenir des nombreuses industries utilisant les ressources vivantes;

veiller à l'utilisation durable des espèces et des écosystèmes (particulièrement les poissons et autres espèces sauvages, les forêts et les pâturages), dont sont tributaires des millions de communautés rurales ainsi que de grandes industries

succession (succession, sucesión) évolution dans le temps de 
la composition d'une espèce dans un écosystème, souvent selon un ordre prévisible (Waring et Schlesinger 1985)

sylviculture (silviculture, silvicultura) théorie et pratique visant à régir l'établissement, la composition, la croissance et la qualité des peuplements forestiers en vue de réaliser certains objectifs d'aménagement (Society of American Foresters 1989)

terre forestière (forest land, terreno forestal) superficie couverte de peuplements d'arbres cultivés ou naturels devant servir à un usage forestier; comprend les terres qui ne comportent plus d'arbres mais doivent être reboisées. Les pelouses et les pâturages permanents comportant des arbres et des arbustes épars ne constituent pas une terre forestière (Maini 1991)

usage polyvalent (multiple use, uso múltiple) conduite de deux activités ou plus (randonnée, chasse, abattage) dans la même zone, a) simultanément dans toute la zone, b) dans des secteurs distincts ou c) successivement

utilisation durable (sustainable use, utilización sostenible) utilisation d'un organisme, d'un écosystème ou d'une autre ressource renouvelable à un rythme qui respecte son potentiel de renouvellement (Alliance mondiale pour la nature et autres 1991)

village planétaire ( $g l o b a l$ village, aldea global) la nouvelle interdépendance électronique recrée le monde à l'image d'un village planétaire. Avec la transmission électrique de l'information à la vitesse de la lumière, tous les événements de la planète sont simultanés et ne sont séparés ni par le temps, ni par l'espace. On peut désormais vivre non seulement dans une région ou dans un pays: on peut vivre simultanément plusieurs cultures dans plusieurs mondes à la fois (McLuhan 1967, McLuhan et Forsdale 1988)

zone humide (wetland, zona anegadiza) zone recouverte d'une eau peu profonde durant certaines saisons ou à l'année, ou zone où la surface de la nappe phréatique est proche de la surface ou affleure. Dans les deux cas, la présence d'eau en abondance a entrainé la formation de sols hydriques et favorisé la prédominance d'hydrophytes ou de plantes hydrophiles (Ministère des Richesses naturelles et ministère des Affaires municipales de l'Ontario 1991)

zone protégée (protected area, zona protegida) zone protégée par une loi, un règlement ou des principes d'exploitation qui visent à limiter la présence ou l'activité de l'homme. La liste qui suit présente huit catégories de zones protégées et leurs objectifs d'aménagement (Alliance mondiale pour la nature et autres 1991)

monument naturel, repère naturel (natural monument,natural landmark; monumento natural, sitio natural) protéger et préserver des réalités naturelles à cause de leur intérêt particulier ou de leurs caractéristiques uniques. Il s'agit d'aires relativement réduites désignées afin de protéger une réalité précise

parc national (national park, parque nacional) protéger les zones naturelles, esthétiques et remarquables d'intérêt national ou international à des fins scientifiques, éducatives et récréatives. Il s'agit d'aires naturelles relativement grandes où l'activité humaine n'a pas eu d'influence majeure et où il est interdit de prélever quoi que ce soit à des fins commerciales paysages terrestres et marins protégés (protected landscapes and seascapes, paisajes terrestres y marinos protegidos) conserver des paysages terrestres ou marins importants qui sont caractéristiques de l'interaction harmonieuse de l'humain, de la terre et de l'eau tout en permettant au public d'en jouir par des activités récréo-touristiques d'une manière intégrée à l'activité économique et au mode de vie normaux de la région. Il s'agit de paysages terrestres ou marins naturels ou artificiels d'une grande beauté où les utilisations traditionnelles du sol demeurent

réserve anthropologique, zone biotique naturelle (anthropological reserve, natural biotic area; reserva antropológica, zona biótica natural) permettre aux sociétés qui vivent en harmonie avec l'environnement de conserver leur mode de vie sans être dérangées par la technique moderne. Cette catégorie est utilisée lorsque des peuples indigènes exploitent les ressources d'une manière traditionnelle

réserve de possibilité (resource reserve, reserva de recur$s o s$ ) protéger les richesses naturelles de la zone pour usage futur et empêcher ou limiter les activités d'exploitation qui pourraient altérer les ressources avant la formulation d'objectifs fondés sur des connaissances et une planification appropriées. Il s'agit d'une catégorie "d'attente" utilisée jusqu'à ce qu'un classement permanent soit possible

réserve naturelle aménagée, réserve naturelle faunique (managed nature reserve, wildlife sanctuary; reserva natural manejada, reserva natural de fauna) assurer les conditions nécessaires pour protéger des espèces, des groupes d'espèces, des communautés biotiques ou des réalités physiques de l'environnement qui présentent une certaine importance, lorsque ceux-ci ont besoin de l'intervention humaine pour continuer d'exister. L'exploitation contrôlée de certaines ressources peut être permise

réserve naturelle intégrale (strict nature reserve, reserva natural integral) protéger la nature et éliminer tout dérangement des phénomènes naturels afin de disposer d'exemples écologiquement représentatifs du milieu naturel à des fins d'étude scientifique, de suivi environnemental et d'éducation et afin de maintenir les ressources génétiques dans un état dynamique et évolutif

zone d'aménagement intégré, zone de ressources aménagées (multiple-use management area, managed resource area; zona de manejo integrado, zona de recursos manejados) préserver les ressources en eau, la production de bois, la vie sauvage, les pâturages et le tourisme; la conservation de la nature vise ici d'abord le soutien des activités économiques (quoique des secteurs particuliers puissent aussi être désignés dans la zone pour atteindre des objectifs de conservation précis).

Il existe deux autres catégories de désignation internationale qui recoupent simultanément plusieurs des huit notions décrites ci-dessus:

réserve de la biosphère (biosphere reserve, reserva de la biosfera) conserver, pour un usage présent et futur, la diversité et l'intégrité des communautés biotiques végétales et animales au sein d'écosystèmes naturels, et sauvegarder la diversité génétique des espèces dont dépend la permanence 
de leur évolution. Il s'agit de lieux désignés internationalement et aménagés pour la recherche, l'éducation et la formation

site du patrimoine mondial (world heritage site, sitio del patrimonio mundial) protéger les réalités naturelles conférant à la zone son importance universelle exceptionnelle

\section{Références}

Aird, Paul L. 1979. Rapport de la réunion consultative sur la stratégie de l'utilisation de la biomasse: Mots de clôture. Ottawa, Ontario, Canada: Comité canadien pour le programme de 1'Unesco sur l'homme et la biosphère.

Aird, Paul L. 1989. "Conservation in a developing nation: The Canadian experience", Facing North/Facing South, Colloquium on Canadian American Relations, Western Michigan University, Kalamazoo, Michigan, États-Unis.

Alliance mondiale pour la nature, Programme des Nations Unies pour l'environnement, Fonds mondial pour la nature. 1991. Caring for the earth: A strategy for sustainable living. Gland, Suisse, Alliance mondiale pour la nature.

Australie, Resource Assessment Commission. 1991. Forest and timber inquiry, draft report, volume 1. Canberra, Australie, Australian Government Publishing Service.

Baker, Richard J. 1989. "Population viability and the Kirtland's Warbler", At the crossroads - extinction or survival, actes du Kirtland's Warbler Symposium, Department of Natural Resources, Lansing (Michigan), États-Unis.

Chambre des Communes du Canada. 1981. Les eaux sournoises: La tragique réalité des pluies acides, Ottawa, Ontario, Canada, rapport du Sous-comité sur les pluies acides du Comité permanent des pêches et des forêts.

Cody, Martin L. 1986. "Diversity, rarity and conservation in Mediterranean-climate regions", dans Soulé, Michael E. (sous la direction de), Conservation biology: The science of scarcity and diversity, Sunderland, Massachusetts, États-Unis, Sinauer Associates Inc.

Commission des communautés européennes. 1983. Glossarium: Nouvelles sources d'énergie, Bruxelles-Luxembourg, Belgique, Bureau de terminologie.

Commission mondiale de l'environnement et du développement. 1988. Notre avenir à tous, Montréal, Éditions du fleuve (Version originale anglaise: Our common future, Oxford, Angleterre, Oxford University Press, 1987.

Conseil international pour la recherche en agroforesterie. 1991. "ICRAF" in the "CGIAR": The way ahead, Nairobi, Kenya, Conseil international pour la recherche en agroforesterie.

Elton, Charles S. 1957. The ecology of invasions by animals and plants, Londres, Angleterre, Methuen and Co. Ltd.

Empire Forestry Association. 1953. British Commonwealth forest terminology: Part I, Londres, Angleterre, Royal Empire Society.

Gilpin, Michael E. et Michael E. Soulé. 1968. "Minimum viable populations: processes of species extinction" dans Soulé, Michael E. (sous la direction de), Conservation biology: The science of scarcity and diversity, Sunderland, Massachusetts, États-Unis, Sinauer Associates Inc.

Groupe de travail sur la faune de l'Ontario. 1991. Face à l'avenir: Une stratégie des espèces sauvages pour l'Ontario, Toronto, Ontario, Canada, ministère des Richesses naturelles.

Hendrickson, Ole. 1993. Direction des sciences et du développement durable, Forêts Canada, Ottawa, Ontario, Canada: communication personnelle.

Jorgensen, Erik. 1986. "Urban forestry in the rearview mirror", Arboreal Journal, 10, pp. 177-190.

Keystone Center. 1991. Final consensus report of the Keystone policy dialogue on biological diversity on federal lands, Keystone, Colorado, États-Unis.
Lacy, Robert C. 1987. "Loss of genetic diversity from managed populations: Interacting effects of drift, mutation, immigration, selection, and population subdivision", Conservation Biology, 1(2), pp. 143-158. Lacy, R., T. Foose, J. Ballou et J. Eldridge. 1992. "Small populations biology and population and habitat viability assessment", Kirtland's Warbler population and habitat viability assessment briefing book, Apple Valley, Minnesota, États-Unis, Captive Breeding Specialist Group, Species Survival Commission, International Union for Conservation of Nature and Natural Resources.

Mace, Georgina M et Russell Lande. 1991. "Assessing extinction threats: Toward a reevaluation of IUCN threatened species categories", Conservation Biology, 5(2), pp. 148-157.

Maini, J.S. 1989. Le développement soutenable et le secteur canadien des forêts, document de travail présenté au Conseil canadien des ministres des forêts, Ottawa, Ontario, Canada, Forêts Canada.

Maini, J.S. 1991. Principes pour l'obtention d'un consensus international sur la conservation et le développement durable de tous les types de forêts du monde, Ottawa, Ontario, Canada, ministère des Forêts. Maini, J.S. et A. Carlisle (sous la direction de). 1974. La conservation au Canada. Aperçu général, Ottawa, Ontario, Canada, Service canadien des forêts, ministère de l'Environnement.

Maini J.S. et O. Ullsten. 1991. Conservation and sustainable development of forests world-wide: Issues and opportunities, Woods Hole, Massachusetts, États-Unis, International Workshop, The Conservation and Utilization of World Forests.

McLuhan, Marshall. 1967. La galaxie Gutenberg, Montréal, Québec, Canada, Éditions Hurtubise HMH ${ }^{\text {tée }}$. (Version originale anglaise: The Gutenberg Galaxy, University of Toronto Press, 1962.

McLuhan, Marshall et Louis Forsdale. 1988. "Technology and the human dimension", Antigonish Review, 74-75, pp. 21-33.

McNeely, Jeffrey A., Kenton R. Miller, Walter V. Reid, Russell A. Mittermeier et Timothy B. Werner. 1990. Conserving the world's biological diversity. Union internationale pour la conservation de la nature et de ses ressources, Gland Suisse, World Resources Institute, Conservation International, World Wildlife Fund-US et Banque mondiale, Washington, D.C., États-Unis.

Ministère de la Culture et des Communications de l'Ontario. 1990. Perspectives du patrimoine: Buts du patrimoine pour l'Ontario, Toronto, Ontario, Canada, Imprimeur de la Reine pour l'Ontario.

Ministère de l'Agriculture du Canada. 1976. Glossaire des termes de la science des sols, Ottawa, Ontario, Canada, Direction de la recherche.

Ministère des Richesses naturelles et ministère des Affaires municipales de l'Ontario. 1991. Déclaration de principes en matière de terres humides: Projet de déclaration de principes pour fins de consultation conformément à l'article 3 de la Loi de 1983 sur l'aménagement du territoire. Toronto, Ontario, Canada.

Mitchell, Bruce. 1986. "The evolution of integrated resource management", dans Lang, R. (sous la direction de), Integrated approaches to resource planning and management, Banff, Alberta, Canada: Banff Centre for Continuing Education.

New Zealand Government. 1991. Resource management act. Wellington Nouvelle-Zélande, loi n ${ }^{\circ} 69$, Government of New Zealand. Peel, A.L. 1991. Forest resources commission: The future of our forests. Victoria, Colombie-Britannique, Canada, Forest Resources Commission. Pimlott, Douglas H. 1971, "Wilderness values in the twentieth century", dans Litteljohn, Bruce M. et Douglas H. Pimlott (sous la direction de), Why Wilderness, New Press, Toronto, Ontario, Canada. Programme des Nations Unies pour l'environnement. 1992. Conference for the adoption of the agreed text of the convention of biological diversity, Nairobi, Kenya, Programme des Nations Unies pour l'environnement.

République des Philippines 1982. Regulations governing the administration, management and disposition of grazing lands, communal grazing lands, and forest lands used for grazing purposes. Diliman, Quezon City, Philippines, Bureau of Forest Development, Ministry of Natural Resources Administrative Order No. 50. 
Sargent, Caroline et M. Lowcock. 1991. "Options for the coordination of international action on forest conservation and management", dans David Howlett et Caroline Sargent (sous la direction de), Technical Workshop to Explore Options for Global Forestry Management, Proceedings, Office of the National Environment Board, Bangkok, Thailande.

Service canadien des parcs. 1991. Politique proposée du Service canadien des parcs, Ottawa, Ontario, Canada. Environnement Canada. Society of American Foresters. 1971, 1983. Terminology of forest science technology practice and products, sous la direction de Robert K. Winters, Washington, D.C., États-Unis.

Society of American Foresters. 1977, 1983. Terminology of forest science technology practice and products, sous la direction de Robert $\mathrm{K}$. Winters, Washington, D.C., États-Unis.

Society of American Foresters. 1989. Recommended changes in silviculture terminology, manuscrit inédit préparé par le Silviculture Instructor's Subgroup, Silviculture Working Group (D2), Bethesda, Maryland, U.S.A.

Tansley, A.G. 1935. "The use and abuse of vegetational concepts and terms", Ecology, 16(3), pp. 284-307.

Tho, Y.P. 1991. "Tropical moist forests — facts and issues", dans David
Howlett et Caroline Sargent (sous la direction de), Technical Workshop to Explore Options for Global Forestry Management, Proceedings, Office of the National Environment Board, Bangkok, Thailande.

Union internationale pour la conservation de la nature et de ses ressources. 1980. Stratégie mondiale de la conservation: La conservation des ressources vivantes au service du développement durable, Gland, Suisse.

Waring, Richard H. et William H. Schlesinger. 1985. Forest ecosystems; concepts and management, Orlando, Floride, ÉtatsUnis, Academic Press Inc.

Wilson, Edward O. 1992. The diversity of life. Cambridge, Massachusetts, États-Unis, Harvard University Press.

World Resources Institute, Union internationale pour la conservation de la nature, Programme des Nations Unies pour l'environnement. 1991. Biodiversity strategy and action plan, Washington D.C., États-Unis, World Resources Institute.

World Resources Institute, Union internationale pour la conservation de la nature et Programme des Nations Unies pour l'environnement. 1992. Global biodiversity strategy: Guidelines for action to save, study, and use earth's biotic wealth sustainably and equitably, Washington, D.C., États-Unis, World Resources Institute. 